\title{
Patterns of spine surgeries at Mulago Hospital
}

J. W. M. Kigera, MBChB, Senior House Officer, Department of Orthopaedics, College of Health Sciences, Makerere University, Kampala, Uganda. Email: jameskigera@yahoo.co.uk

\section{ABSTRACT}

Background: Spine surgery is a specialised area of orthopaedics that is still in its formative stages in Africa. It may be done to relieve symptoms, or stabilise the spine to allow rehabilitation of patients. This review analyses spine surgeries done in the period 2005-2009 in a National Referral Hospital.

Objectives: Patterns of spine surgeries.

Design: A retrospective review of patients who underwent spine surgeries.

Setting: Mulago National Referral Hospital, Uganda.

Methods: Data was collected from theatre logs and the patient files retrieved for perusal. The data was then analysed.

Results: Spine surgeries have been increasing in number over the years with a marked increase in 2009. Male patients were predominantly due to trauma while females were usually due to degenerative causes.

Conclusion: Trauma especially that caused by road traffic accidents needs a holistic approach to adequately manage. There is need to facilitate surgeons with training and equipment so that their output may increase. Trauma still predominates as an indication for spine surgery. Outcome may be affected by delays in referral to the spine unit. The spine surgeon is increasingly being called upon to managed degenerative and developmental conditions.

\section{INTRODUCTION}

Spinal conditions are associated with great morbidity. Spine surgery is done to prevent further damage to the contents of the spinal canal, relieve symptoms or to stabilize the spine to allow rehabilitation. This is done by ensuring spinal alignment and stability (1).Spine surgery is a specialized area of orthopaedics and is still in its infancy in East Africa. While trauma greatly overwhelms surgeons in Africa, we are more and more being called upon to manage degenerative and developmental conditions. Mulago Hospital is the National Referral Hospital as well as the teaching hospital for Makerere University.The Spine Unit is the only of its kind in the country and inevitably gets referrals from all over the country and beyond especially from the great lakes region. In February 2009, a dedicated spine theatre was opened and operates on three days every week with an average of six cases every week.

Documenting the cases seen and operated on will help the surgeons in this region to understand the different types of spinal pathologies one is likely to encounter.It also helps to document the challenges encountered in trying to establish a spine service in the developing world and share experiences on ways to mitigate these challenges. This retrospective review analysed the spine surgeries done, the indications and anatomical level.

\section{MATERIALS AND METHODS}

This retrospective study included all patients who underwent an operative procedure for a spine condition by the orthopaedic surgical team in the period 2005 - 2009. The patients were identified from the theatre logs and the files retrieved for perusal. Patient files were perused for the demographics, diagnosis and operative procedure done. The data was then analysed. The diagnoses were categorised to aid analysis. The level of the lesion was determined and categorised. Injuries spanning the cervical and thoracic regions were labeled cervico-thoracic while those spanning the thoracic and lumbar regions and the lumbar and sacral regions thoraco-lumbar and lumbo-sacral respectively. 


\section{RESULTS}

A total of 158 surgeries were performed in various theatres in Mulago in the period under review. The number of surgeries has been increasing over the years. The dramatic increase in the year 2009 is attributed to the commissioning of a dedicated spinal unit theatre (Figure 1).

Male patients constituted $60.8 \%(n=96)$ with a male to female ratio of 1.5:1.

The youngest patient was four years old while the oldest was 78 years while the mean age was 37.2 years (Figure 2). Female patients were clustered in the 30 to 50 years age groups with a mean age of 40.1 years while the male patients tended to be younger with a mean age of 36.5 years (Figures 3 and 4).

Majority of the patients had trauma as the diagnosis (43\%) while the second most common classification was degenerative diseases at 26\% (Figure 5).

The lumbar spine was most involved followed by the cervical and the thoracic spine. The junction areas were infrequently involved (Figure 6).

There was a wide variety of procedures done. The most common procedures done were posterior lumbar decompression with instrumentation and fusion (Table 1)

The duration of stay ranged from 23 days to 133 days with a mean of 51 days. The duration of stay after surgery ranged from 5 days to 124 days with a mean of 29 days.

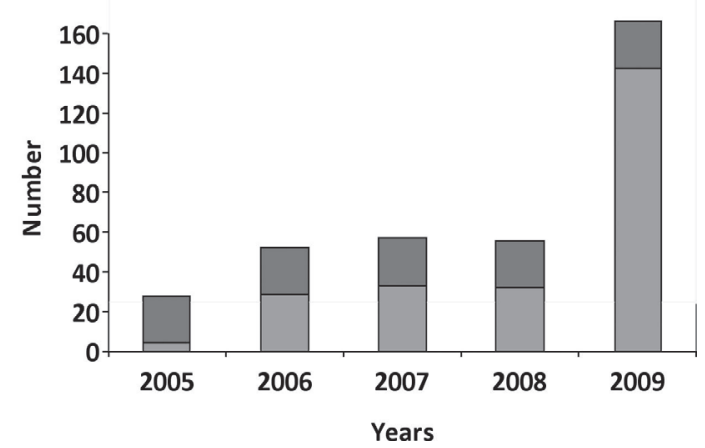

Figure 1: Surgeries done in each year

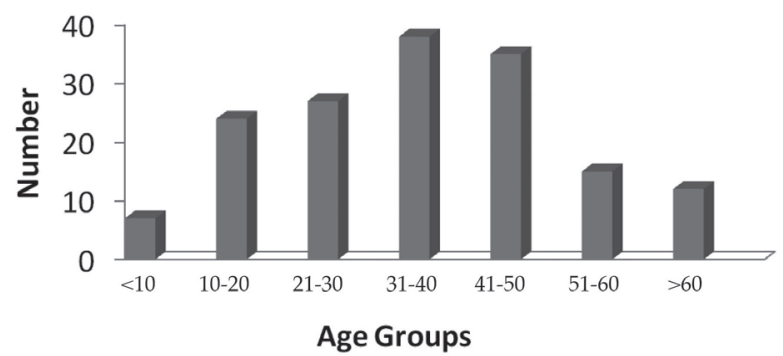

Figure 2: Age distribution

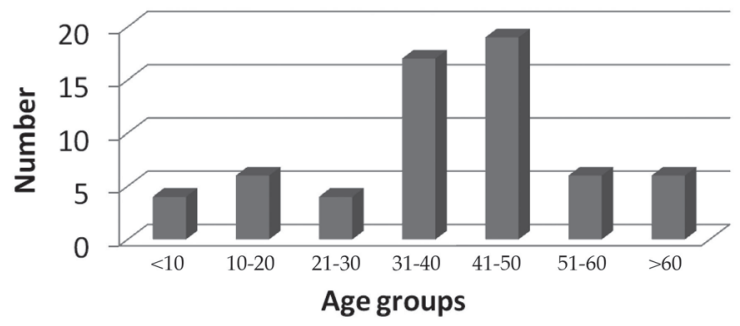

Figure 3: Age distribution in females

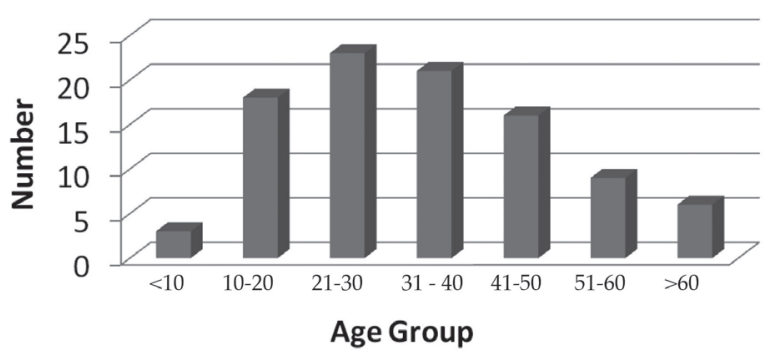

Figure 4: Age distribution in males

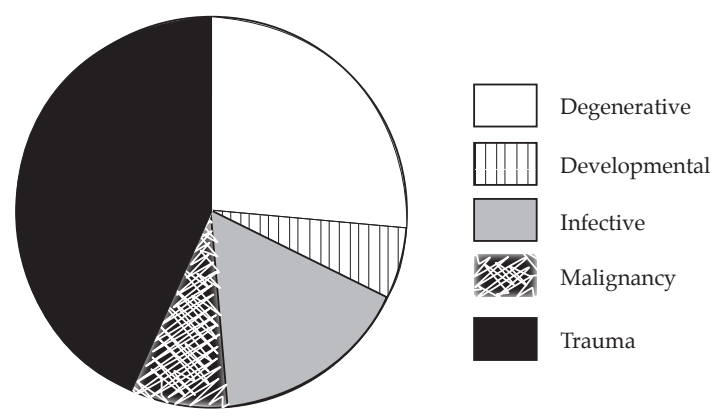

Figure 5: Classification of diagnoses

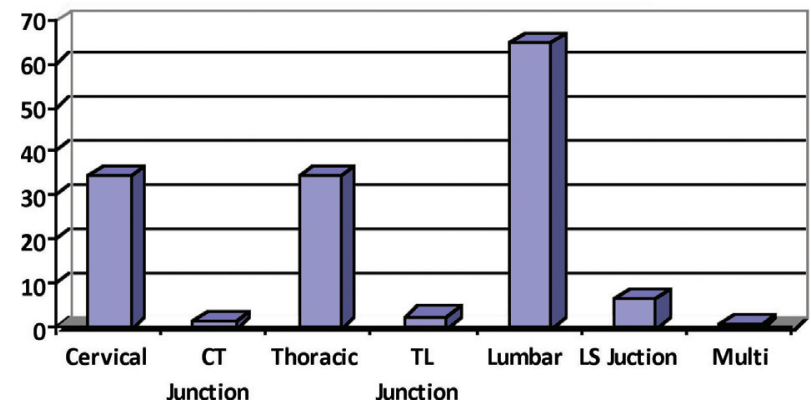

Figure 6: Anatomical levels 
Table 1

Procedures done

\begin{tabular}{lc}
\hline Procedures done & Number \\
\hline Posterior lumbar decompression, instrumentation and fusion & 40 \\
Posterior lumbar decompression, instrumentation and fusion & 31 \\
Anterior cervical decompression, instrumentation and fusion & 24 \\
Open biopsy & 17 \\
Anterior thoracic decompression/release and fusion & 15 \\
Posterior cervical decompression and fusion & 11 \\
Costotransversectomy & 7 \\
Anterior lumbar decompression and Fusion & 5 \\
Posterior thoracic release and fusion & 4 \\
Image guided biopsy & 2 \\
Implant removal & 1
\end{tabular}

\section{DISCUSSION}

The epidemiology of the patients is varied. Most of the patients with degenerative diseases are female while males dominated the trauma category (2). Trauma is a leading cause as has been seen in other African situations. The male predominance in trauma cases has been noted by many investigators. Spinal trauma is known to affect young men with grave socio economic consequences to them and their families (3).

There is a delay in presentation of trauma patients and this may affect the quality of care given and the eventual outcome. This has been noted by other investigators and has been attributed to the long distances that need to be covered from the peripheral centers and inadequate bed space in spine units (3). This affects morbidity and prognosis. Medical workers in peripheral centers need to be sensitized on the need for quick referrals. The long duration of stay after operation highlights the difficulties in rehabilitation of these patients. There is need to improve the rehabilitation facilities and probably have a separate centre for this purpose. The belief in traditional bone setters cannot be discounted as a reason for delayed referral.The practice of traditional bone setters is rife in sub-Saharan Africa and severe complications have been encountered.

In centers with a high demand for spine surgical services, early surgery has been shown to reduce morbidity and mortality with increased throughput (3).

The average duration of stay of 51 days was unacceptably high. Other investigators report durations of about 32 days. This could be due to the fact that our spine unit doubles as the rehabilitation centre while most centers discharge their patients to separately housed rehabilitation centers (3).

\section{CONCLUSIONS}

Spine surgery has grown over the years and there is need to further develop this expert area of orthopaedics. Spine surgeons need to be facilitated with the necessary resources and their output will greatly improve.

Trauma contributes majority of cases operated and efforts need to be made to reduce the trauma especially on our roads. More efforts and expertise are needed in the area of spine rehabilitation. This can be in training of personnel and better and provision of more equipped rehabilitation facilities.

\section{REFERENCES}

1. Stauffer, E.S. Subaxial injuries. Clin. Orthop. Rel. Res. 1989; 30: 239.

2. Emejulu, J.K.C. and Ekweogwu, O.C. and Nottidge, T. Patterns of spinal injury in a new neurosurgical centre: A 2-year prospective study. East and Central Afr. J. Surg. 2009; 14:76-80.

3. Frielingsdorf, K. and Dunn, R. N. Cervical spine injury outcome - a review of 101 cases treated in a tertiary referral unit. SAMJ. 2007; 97: 203-207.

4. Idowu, O. E. Idika, O. C. and Apemiye, R. A. Cervical myelopathy from traditional bonesetters' treatment of spinal injury. East and Central Afr. J. Surg. 2008; 10: 77-78. 